\title{
Corpus allatum volume-dependent differences in accessory gland maturation in long- and short-winged males of Pyrrhocoris apterus (Heteroptera: Pyrrhocoridae)
}

\author{
RAdOMÍR SOCHA ${ }^{1}$ and Magdalena HODKOVÁ ${ }^{1,2}$ \\ ${ }^{1}$ Institute of Entomology, Academy of Sciences and ${ }^{2}$ Faculty of Biological Sciences, University of South Bohemia, \\ Branišovská 31,370 05 České Budějovice, Czech Republic; e-mail: socha@entu.cas.cz
}

Key words. Corpus allatum, accessory glands, wing morphs, adult males, Pyrrhocoris apterus

\begin{abstract}
In the present study the temporal changes in the volume of the corpus allatum in three experimental groups of adult males (macropterous, reproductive brachypterous and diapausing brachypterous) of the flightless bug Pyrrhocoris apterus were determined and related to the size of male accessory glands. The results revealed wing morph- and age-dependent differences in the corpus allatum volume in males of this bug. In 4-14 day old males, the volumes of the corpus allatum and accessory glands were largest in long-day reproductive brachypters, intermediate in long-day macropters, and smallest in short-day diapausing brachypters. The smaller corpus allatum in young macropterous males than in same aged reproductive brachypterous males was due to the spontaneous fasting of the former. Later, starting on day 18 after adult emergence, i.e. when macropterous males were feeding normally, there were no significant differences in the volumes of the corpus allatum between long-day brachypterous and macropterous males. On the other hand, the corpus allatum of 18-28 day old diapausing brachypterous males was significantly smaller than that of same aged long-day macropterous and reproductive brachypterous males. The sizes of the corpus allatum and accessory glands were significantly positively correlated in macropterous and diapausing brachypterous males. This is the first report of corpus allatum volume-dependent wing morph-related differences in the rate of accessory gland maturation in males of insects with a non-functional macropterism. The role of differential activity of the corpus allatum in the different life history strategies of males of the two wing morphs in this wing-polymorphic insect is discussed.
\end{abstract}

\section{INTRODUCTION}

The firebug Pyrrhocoris apterus (L.) is a typical representative of wing-polymorphic insects that have evolved flightlessness (Honěk, 1995; Socha \& Zemek, 2000a). It is a common Palaearctic phytophagous species producing individuals of long-winged (macropterous) and shortwinged (brachypterous) morphs (Socha, 1993). In this bug, both reproductive diapause and wing polymorphism are controlled by photoperiod and temperature (Hodek, 1968; Honěk, 1976; Socha, 2001). The development of the macropterous morph is controlled by a recessive allele, which is expressed under long-day and high temperature conditions (Honěk, 1976, 1986). Under short-day conditions (photophase $<16 \mathrm{~h}$ ), bugs from Czech populations go into diapause and virtually all are brachypterous, while under longer days they do not enter diapause and a fraction of the population (0-14\% in the populations from the Czech Republic) becomes macropterous (Honěk, 1976, 1981). Macropterous individuals of P. apterus are flightless (Socha \& Zemek, 2000a) and differ from brachypters in various physiological and behavioural parameters, e.g. longer pre-oviposition period (Honěk, 1985; Socha \& Šula, 1996), lower feeding and digestive enzyme activities (Socha et al., 1997, 1998), higher adipokinetic response (Socha \& Kodrík, 1999) and enhanced dispersal by walking (Socha \& Zemek, 2000b, 2003). An increase in the speed of locomotion of macropters is positively correlated with a more intensive mobilization of lipids from the fat body by the adipoki- netic hormone (AKH) and a higher content of this hormone in the corpora cardiaca and brain (Maxová et al., 2001; Kodrík et al., 2003). This accords with the fact that AKHs are usually synthetized and stored by neurosecretory cells of the corpora cardiaca and predominantly involved in the control of energy metabolism (for review see Gäde, 2004). The above data indicate that the two wing morphs of $P$. apterus differ in their life history strategies.

Recent studies (Socha, 2004; Socha \& Zemek, 2004a,b) have shown that the greater tendency of flightless macropters of $P$. apterus to disperse is associated with lower mating activity, as in many wing-polymorphic insects that can fly (Zera \& Denno, 1997). Macropterous pairs of this bug copulated almost three times less frequently than brachypterous pairs (Socha \& Zemek, 2004a). The lower mating propensity of macropterous pairs was mainly due to the lower receptivity of females associated with an inhibition of ovarian growth and to some extent the lower mating vigour of the males (Socha \& Zemek, 2004b). Receptivity tests revealed that reproductive brachypterous females have a higher tendency to mate than macropterous females (Socha, 2004). Similar differences in mating activity are also recorded for reproductive brachypterous and macropterous males (Socha, 2004). Long-day brachypterous males are two to four times more successful in competing for females than macropterous males, depending on the wing morph and physiological status of the females (Socha, 2004). Higher competitive ability of long-day brachypterous males is associated with 
larger accessory glands (AGs) (Socha, 2006). Elevation of total proteins in AGs and an increase in the mating success of macropterous males of $P$. apterus treated with juvenile hormone analogue ( $\mathrm{JHa}$ ) on the level observed in reproductive brachypterous males (Socha et al., 2004; Socha, 2006), indicate that wing morph-related difference in the rate of accessory gland maturation in adult males is dependent on differences in the activity of the corpus allatum (CA) - the source of juvenile hormone (JH). However, direct evidence of this possibility has not yet been documented.

In the present study the hypotheses that (1) the temporal changes in the volume of the CA in long-day macropterous, long-day brachypterous and short-day brachypterous adult males differ, and (2) there is a relationship between the temporal changes in CA volume and the size of AGs in adult males, were tested.

\section{MATERIAL AND METHODS}

\section{Experimental animals}

The stock cultures of Pyrrhocoris apterus, which produced the brachypterous and macropterous adults (Socha \& Šula, 1997, 1998) were used in the present study. They originated from a wild population in České Budějovice, Czech Republic $\left(49^{\circ} \mathrm{N}\right)$. Larvae and adults were kept in mass culture in 0.51 glass jars (approximately 40 specimens per jar) and supplied with linden seeds and water in glass tubes plugged with cellulose wool. The food and water were replenished twice a week. Three experimental groups of adult males (macropterous, reproductive brachypterous and diapausing brachypterous) were used in the present study. The macropterous (M) and reproductive brachypterous (B-R) bugs were maintained under a long-day photoperiod of $18 \mathrm{~L}: 6 \mathrm{D}$ and a constant temperature of $26 \pm$ $1^{\circ} \mathrm{C}$, while the diapausing brachypterous (B-D) bugs were obtained from a culture in which the bugs were reared from eggs to adults under a short-day photoperiod of $12 \mathrm{~L}: 12 \mathrm{D}$ and the same temperature (Hodek, 1968). Freshly ecdysed adult males were removed from the cultures, separated according to wing morph and physiological status (M, B-R, B-D), and each of these experimental groups kept separately in small glass jars (0.25 1) in groups of $10-15$ specimens. They were kept in the same photoperiodic regimen as they developed. When these adults reached a particular age, they were individually used for the following experiments.

To avoid the possible effect of the rhythmic changes, all the measurements of $\mathrm{CA}$ volume and $\mathrm{AG}$ size were made at the same time of day, 10-11 h a.m, i.e. 3-4 h after the light was switched on. This time was chosen on the basis of previous studies that indicated that the maxima in walking (Socha \& Zemek, 2000b) and mating (Socha \& Zemek, 2004b) activities, and highest content of AKH (Kodrík et al., 2003) and intensity of the adipokinetic response (Maxová et al., 2001) in P. apterus adults occurred during the photophase, irrespective of wing morph.

\section{Determination of corpus allatum volume}

All the measurements were made using a stereomicroscope with an ocular micrometer attachment. The volume of the CA was estimated using the method described by Hodková (1977b). The CA volume was measured in approximately 10 individuals of $1,2,3,4,5,6,7,8,9,10,14,18$ and 28 days old M, B-R and $\mathrm{B}-\mathrm{D}$ males and the values averaged.

\section{Measurement of the size of the accessory glands}

The AGs were first dissected under isotonic insect Ringer saline from males with the aid of an Olympus binocular microscope. Then, the AGs were placed on microscope slides in a small drop $(10 \mu \mathrm{l})$ of isotonic insect Ringer saline and measured using an ocular micrometer. Three different parameters of AG size (length, height and width) were measured, but as the shape of the glands was the same throughout adult development, only the length data are presented in this paper. The size of AGs was measured in at least 10 individuals of $1,3,5,7,10,14,18$ and 28 days old B-D males and the values averaged. Temporal changes in AG size of B-D males were compared with those for $\mathrm{M}$ and B-R males obtained by Socha et al. (2004).

\section{Analysis of the relationship between CA volume and AG size}

At this stage of the research the relationship between CA volume and size of AGs and whether temporal changes in this endocrine gland volume might reflect the rate of maturation of these reproductive glands were studied. A logistic regression analysis of the present and previous data (Socha et al., 2004) was used to determine the relationship between CA volume and AG size.

\section{Data presentation and statistical analyses}

The graphs and statistical evaluation of the results were made using the software Graph Pad Prism, version 4.0 (San Diego, CA, USA). The values for CA volumes and AG sizes were analysed using a one-way ANOVA with Bonferroni's Multiple Comparison test. A logistic regression analysis was used to determine the relationship between CA volume and AG size for the males in the different experimental groups.

\section{RESULTS}

\section{Age- and wing morph-related changes in the corpus allatum volume}

The temporal changes in the volumes of CA dissected from males of the three experimental groups are presented in Fig. 1. The largest CA volume was recorded for B-R males, the smallest for B-D males. Despite the similar rhythm in the increase and decrease in CA volume in B-R and $M$ males, the CA volume of 4-14 day old $M$ males had an intermediate value between that for same aged $\mathrm{B}-\mathrm{R}$ and $\mathrm{B}-\mathrm{D}$ males. However, the $\mathrm{CA}$ volume in $\mathrm{M}$ males was significantly smaller $(P<0.05)$ and larger $(P<$ $0.01)$ than in B-R and B-D males of the same age, respectively, only between 7-14 days after adult emergence. After 18 days there was no significant difference between the CA volumes of $\mathrm{M}$ and B-R males. On the other hand, the volume of the CA from 18-28 day old B-D males was significantly lower $(P<0.001)$ than that of same aged $\mathrm{M}$ and B-R males.

\section{Age- and wing morph-related changes in the accessory gland size}

At this stage of the research the differences in the temporal pattern of AG size in males of the three experimental groups were studied. For this purpose, the AG sizes of the B-D males in the present study were compared with those of $\mathrm{M}$ and B-R males obtained in our earlier study (Socha et al., 2004) and the combined data were presented in Fig. 2. Comparison of the AG sizes revealed similar relations among the sizes of AGs of B-R, B-D and $\mathrm{M}$ males as among the volumes of their CA. The B-R 


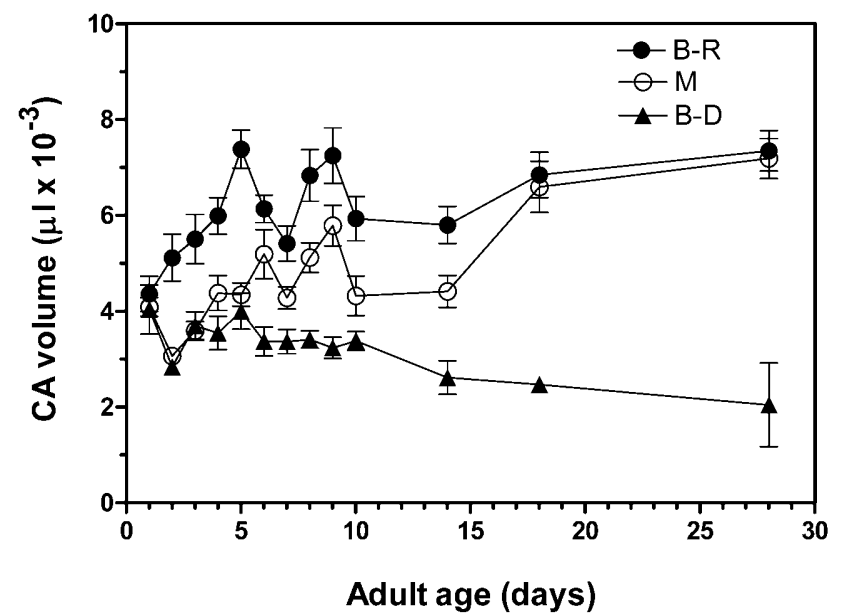

Fig. 1. Temporal changes in the volume of the corpus allatum in macropterous, and reproductive and diapausing brachypterous males of $P$. apterus during the first 28 days of adult life. Abbreviations: $\mathrm{M}$ - macropterous; $\mathrm{B}-\mathrm{R}$ - reproductive brachypterous; B-D - diapausing brachypterous. Vertical lines indicate the means \pm S.E; each mean is based on at least 10 adult males.

males had the largest AGs, B-D males the smallest, whilst that of 3-14 day old $M$ males was intermediate between that for B-R and B-D males. The AG size of 3-14 day old $\mathrm{M}$ males was significantly lower $(P<0.05-0.001)$ and higher $(P<0.001)$ than that of same aged B-R and B-D males, respectively. From day 18 onwards there was no significant difference between the AG size of $\mathrm{M}$ and B-R males. As for CA volume, the size of AGs in 18-28 day old B-D males was significantly $(P<0.001)$ lower than in same aged $\mathrm{M}$ and $\mathrm{B}-\mathrm{R}$ males.

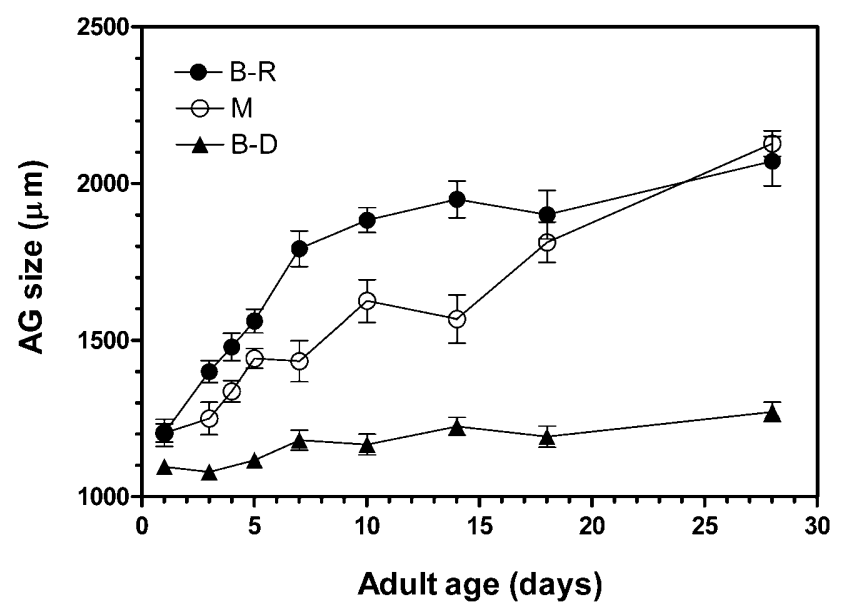

Fig. 2. Temporal changes in the size of accessory glands in macropterous, and reproductive and diapausing brachypterous males of $P$. apterus during the first 28 days of adult life. Abbreviations: $\mathrm{M}$ - macropterous; $\mathrm{B}-\mathrm{R}$ - reproductive brachypterous; B-D - diapausing brachypterous. Vertical lines indicate the means \pm S.E; each mean is based on at least 10 adult males. The AG sizes for M- and B-R males were taken from Socha et al. (2004).
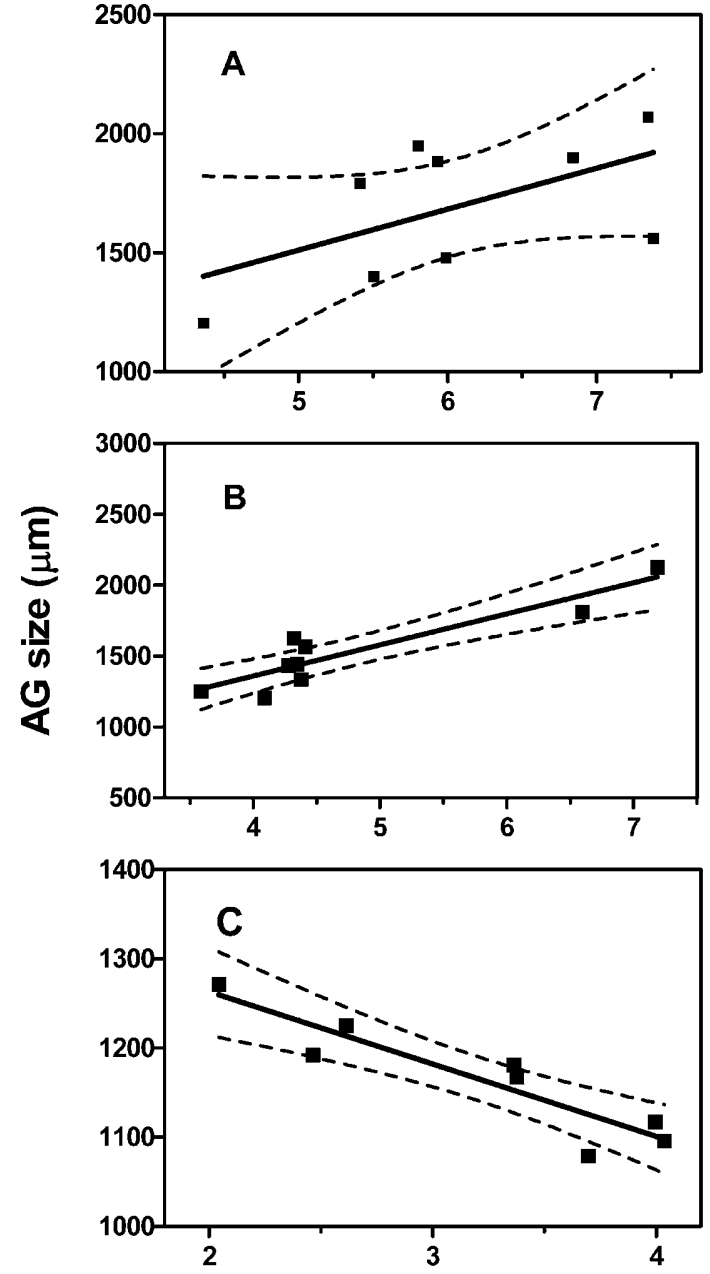

CA volume $\left(\mu 1 \times 10^{-3}\right)$

Fig. 3. Relationship between the volume of the corpus allatum and size of accessory glands in macropterous (A), and reproductive (B) and diapausing (C) brachypterous males of $P$. apterus during the first 28 days of adult life. Abbreviations: $\mathrm{M}-$ macropterous; B-R - reproductive brachypterous; B-D - diapausing brachypterous. Solid line, regression $\left(\mathrm{A}: r^{2}=0.8363, P\right.$ $=0.0006 ; \mathrm{B}: r^{2}=0.3332, P=0.1037 ; \mathrm{C}: r^{2}=0.8399, P=$ $0.0014)$; dashed lines, $95 \%$ confidence intervals.

\section{Relationship between corpus allatum volume and accessory gland size}

The next question was whether the CA volume reflected the size of the AG. A logistic regression analysis of the present (volume of CA in M, B-R and B-D males and $\mathrm{AG}$ size of B-D males) and previously published data, including the AG size of $\mathrm{M}$ and B-R males (Socha et al., 2004), revealed a significant positive relationship between the $\mathrm{CA}$ volume and $\mathrm{AG}$ size in $\mathrm{M}(P=0.0006)$ and B-D males $(P=0.0014)$, but not in B-R males $(P=$ 0.1037) (Fig. 3). The absence of a significant positive relationship in B-R males is due to one extremely high value of $\mathrm{CA}$ volume on day 5 , because removing this value from the linear regression analysis results in a significant positive correlation $\left(r^{2}=0.6053, P=0.0230\right)$ between $\mathrm{CA}$ volume and AG size in these males. A posi- 
tive relationship between $\mathrm{CA}$ volume and size of the $\mathrm{AG}$ in $M$ males indicates that the delay in the maturation of the AGs in the males of this wing morph is associated with the lower volume of their CA, which is intermediate between the values recorded for B-R and B-D males.

\section{DISCUSSION}

\section{The corpus allatum volume vs. accessory gland size in $P$. apterus males}

The largest CA and AGs were recorded in long-day $\mathrm{B}-\mathrm{R}$ males, intermediate sized ones in long-day $\mathrm{M}$ males and the smallest in short-day B-D males. In P. apterus, the synthetic activity of the CA in vitro is well correlated with CA size. No activity was detected in small CA of short-day diapausing brachypterous females, in which the growth of vitellogenic ovaries is inhibited. The activity of the larger CA of long-day reproductive females with vitellogenic ovaries is about four times higher than that of the CA of intermediate size from starving long-day brachypterous females with pre-vitellogenic ovaries (Hodková et al., 2001). Considering the above facts, the results obtained from the present study indicate that differences in the size and rate of maturation of AGs among M, B-R and B-D males of P. apterus are also associated with different levels of CA activity. The slower maturation of AGs in spontaneously fasting $\mathrm{M}$ males ( $<18$ days old) than in the same aged B-R males appears to be a result of the significantly smaller volume and lower activity of the CA in the former, as in artificially starved long-day brachypterous females. The smaller CA volume in short-day B-D males than in spontaneously fasting long-day $\mathrm{M}$ males accords well with the findings that the CA of brachypterous diapausing adults is inactive (Sláma, 1964) and the CA of short-day diapausing brachypterous females of $P$. apterus is also smaller than that of artificially starved long-day brachypterous females (Hodková, 1999). The CA volume-dependent size of AGs observed in the present study is also supported by the finding that allatectomy of long-day brachypterous males results in a reduction in AG size, while the treatment of allatectomized brachypterous males with an active JHa restores the AG size to the level typical for normally feeding B-R males (Socha, 2006). Enlargement of AGs in longwinged males after JHa treatment is also reported for the flying cricket Modicogryllus confirmatus (Tanaka, 1999). Thus, in P. apterus, as in many other insects, the maturation of AGs and the synthesis of their secretory proteins are JH-dependent processes (Chen, 1984; Happ, 1992; Wolfner, 2002; Gillott, 2003; Kubli 2003; Socha et al., 2004).

The lower activity of the CA in young spontaneously fasting M males (Socha et al., 2001) is most probably due to nervous inhibition of the CA, as in short-day and artificially starved long-day brachypterous females of $P$. apterus (Hodková, 1976, 1977a, 1982). This accords with the observation that continued starvation of heteropteran adults leads to cessation of allatal activity and causes $\mathrm{JH}$ depletion (Johansson, 1958; Rankin \& Riddiford, 1977), and that starved females of the cockroach Blattella ger- manica also do not increase CA volume and produce $\mathrm{JH}$ at very low rates (Osorio et al., 1998). The subsequent increase in $\mathrm{CA}$ volume and $\mathrm{AG}$ size in older $\mathrm{M}$ males of $P$. apterus ( $>18$ days old) to the level typical for normally feeding B-R males most probably resulted from renewed feeding. It cannot be excluded that the two smaller peaks in the temporal pattern of the changes in CA volume in B-R and $M$ males that occurred 5-6 and 9 days after adult ecdysis might represent rhythmical changes in endocrine gland volume associated with those in feeding activity. It is known that food intake stimulates the growth and gonadotropic activity of the $\mathrm{CA}$ in long-day brachypterous females of $P$. apterus and that the synthetic activity of the CA increased only if it was connected with the brain during feeding (Hodková, 1999). Feeding serves as a direct trigger removing the inhibition of CA and the stimulation due to feeding can be transmitted to a transplanted brain-corpora cardiaca-corpus allatum complex via the haemolymph (Hodková, 1982, 1992; Hodková et al., 2001). However, in most heteropteran species, the nature of the humoral factor that mediates the effect of feeding has yet to be identified. In Rhodnius prolixus, the feeding stimulus is transmitted to the brain via a peptide hormone originating in the thoracic ganglionic mass (Mulye \& Davey, 1995).

\section{Endocrine control of wing morph-related differences in life history strategies of $\boldsymbol{P}$. apterus males}

The present and previous (Socha et al., 2004) studies indicate that it is the lowered activity of the CA coupled with a decrease in $\mathrm{JH}$ content in the body that is responsible for the slower maturation of AGs in M males of $P$. apterus, their delayed sexual maturity and lower mating success. As in starved males of the bug Dysdercus koenigii (Hebbalkar \& Sharma, 1982), the smaller size of AGs and reduced mating activity of the young fasting $M$ males of P. apterus (Socha, 2004) resulted from the suppression of CA activity. This suggestion is well supported by recent findings that both the rate of $A G$ maturation and the mating success of $\mathrm{M}$ males of $P$. apterus can be significantly increased by JHa treatment (Socha, 2006). Thus, AG size is an important critical determinant of sexual maturity and the level of mating activity in $P$. apterus males, as in two wing monomorphic species, the fruit fly Drosophila melanogaster (Bangham et al., 2002) and stalk-eyed fly, Cyrtodiopsis dalmanni (Baker et al., 2003). JH plays an important role in the regulation of sexual maturation and/or mating behaviour in the males of some other insect species, e.g. locusts Schistocerca gregaria (Loher, 1961), Locusta migratoria (Wajc \& Pener, 1969) and L. septemfasciata (Pener, 1968) and the bug D. koenigii (Hebbalkar \& Sharma, 1982). Despite the fact that allatectomy and starvation do not affect the development of sexual instincts in long-day brachypterous males of $P$. apterus (Žd'árek, 1970), the present and the most recent (Socha et al., 2004; Socha, 2006) studies indicate an involvement of $\mathrm{JH}$ in differential mating success of macropterous and brachypterous males of this bug. This accords with the finding that $\mathrm{JH}$ is involved in the mating behaviour of $D$. melanogaster 
males, either directly or indirectly, through a male accessory gland component (Wilson et al., 2003). It is known that components in male seminal fluid play a significant role in the reproductive success of various insects (for reviews see e.g. Wolfner, 2002; Gillott, 2003; Chapman \& Davies, 2004).

It is probable that the low sexual activity and high walking activity of young M males ( $<18$ days old) (Socha $\&$ Zemek, 2003; Socha, 2004) are associated with a low activity of the CA (present results) and an increased titre of AKH in the body (Socha et al., 2005). It appears that both $\mathrm{JH}$ and $\mathrm{AKH}$ coordinate reproduction and dispersal in flightless macropterous individuals of $P$. apterus. The latter hormone mobilizes lipid reserves from the fat body to supply the energy for the enhanced walking activity of macropterous individuals (Socha et al., 1998; Kodrík et al., 2000). Mobilization of lipids from the fat body and enhanced walking activity of macropterous adults of $P$. apterus can be stimulated by the injection or topical application of AKH (Socha et al., 1999; Kodrík et al., 2002). In the milkweed bug Oncopeltus fasciatus, however, it is the $\mathrm{JH}$ that is primarily responsible for stimulation and coordination of migratory flight and reproduction, while the corpus cardiacum does not seem to be involved in migratory behaviour of this species (Rankin \& Riddiford, 1977).

Considering all the above findings, it appears that the different activity of the CA and titre of AKH in the body play an important and probably the main role in the wing morph-related differences in the reproductive and dispersal strategies of $P$. apterus males.

ACKNOWLEDGEMENTS. This study was supported by grant No. A6007301 from the Grant Agency of the Academy of Sciences of the Czech Republic (RS). The authors thank J. Mikešová and D. Rienesslová for their technical assistance.

\section{REFERENCES}

Baker R.H., Denniff M., Futerman P., Fowler K., PomianKowski A. \& Chapman T. 2003: Accessory gland size influences time to sexual maturity and mating frequency in the stalk-eyed fly, Cyrtodiopsis dalmanni. Behav. Ecol. 14: 607-611.

Bangham J., Chapman T. \& Partridge L. 2002: Effects of body size, accessory gland and testis size on pre- and postcopulatory success in Drosophila melanogaster. Anim. Behav. 64: 915-921.

Chapman T. \& Davies S.J. 2004: Functions and analysis of the seminal fluid proteins of male Drosophila melanogaster fruit flies. Peptides 25: 1477-1490.

CHEN P.S. 1984: The functional morphology and biochemistry of insect male accessory glands and their secretions. Annu. Rev. Entomol. 29: 233-255.

GäDE G. 2004: Regulation of intermediary metabolism and water balance of insects by neuropeptides. Annu. Rev. Entomol. 49: 93-113.

GiLLot C. 2003: Male accessory gland secretions: Modulators of female reproductive physiology and behavior. Annu. Rev. Entomol. 48: 163-184.

HAPP G.M. 1992: Maturation of the male reproductive system and its endocrine regulation. Annu. Rev. Entomol. 37: 303-320.
Hebbalkar D.S. \& Sharma R.N. 1982: An experimental analysis of mating behaviour of the bug Dysdercus koenigii F. (Hemiptera: Pyrrhocoridae). Indian J. Exp. Biol. 20: 395-405.

Hodek I. 1968: Diapause in females of Pyrrhocoris apterus L. (Heteroptera). Acta Entomol. Bohemoslov. 65: 422-435.

Hodková M. 1976: Nervous inhibition of corpora allata by photoperiod in Pyrrhocoris apterus. Nature 263: 521-523.

HodKová M. 1977a: Nervous pathways in photoperiodic regulation of reproduction in females of Pyrrhocoris apterus (Hemiptera). Acta Entomol. Bohemoslov. 74: 353-361.

HodKová M. 1977b: Size and gonadotropic activity of corpus allatum after different surgical treatments in Pyrrhocoris apterus females (Heteroptera). Věst. Čs. Spol. Zool. 41: 8-14.

HodKová M. 1982: Interaction of feeding and photoperiod in regulation of the corpus allatum activity in females of Pyrrhocoris apterus L. (Hemiptera). Zool. Jb. Physiol. 86: 477-488.

HodkovÁ M. 1992: Storage of the photoperiodic "information" within the implanted neuroendocrine complexes of the linden bug Pyrrhocoris apterus L. (Heteroptera). J. Insect Physiol. 38: $357-363$.

Hodková M. 1999: Regulation of diapause and reproduction in Pyrrhocoris apterus (L.) (Heteroptera) - neuroendocrine outputs (mini review). Entomol. Sci. 2: 563-566.

Hodková M., Okuda T. \& Wagner R.M. 2001: Regulation of corpora allata in females of Pyrrhocoris apterus (Heteroptera) (a mini-review). In Vitro Cell. Dev. Biol. Anim. 37: 560-563.

HoNĚK A. 1976: The regulation of wing polymorphism in natural populations of Pyrrhocoris apterus (Heteroptera, Pyrrhocoridae). Zool. Jb. Syst. 103: 547-570.

HoNĚK A. 1981: Temperature and wing polymorphism in natural populations of Pyrrhocoris apterus L. (Heteroptera, Pyrrhocoridae). Zool. Jb. Syst. 108: 487-501.

HoNĚK A. 1985: Ecophysiological differences between brachypterous and macropterous morphs in Pyrrhocoris apterus (Heteroptera, Pyrrhocoridae). Acta Entomol. Bohemoslov. 82: 347-354.

HoNĚK A. 1986: Inheritance of wing form in Pyrrhocoris apterus. J. Hered. 77: 465-467.

HoNĚK A. 1995: Factors and consequences of a non-functional alary polymorphism in Pyrrhocoris apterus (Heteroptera: Pyrrhocoridae). Res. Popul. Ecol. 37: 111-118.

JoHANSSON A.S. 1958: Relation of nutrition to endocrinereproductive functions in the milkweed bug Oncopeltus fasciatus (Dallas) (Heteroptera: Lygaeidae). Nytt. Mag. Zool. 7: $1-132$.

Kodrík D., Socha R., Šimek P., Zemek R. \& Goldsworthy G.J. 2000: A new member of the AKH/RPCH family that stimulates locomotory activity in the firebug, Pyrrhocoris apterus (Heteroptera). Insect Biochem. Mol. Biol. 30: 489-498.

Kodrík D., Socha R. \& Zemek R. 2002: Topical application of Pya-AKH stimulates lipid mobilization and locomotion in the flightless bug, Pyrrhocoris apterus (L.) (Heteroptera). Physiol. Entomol. 26: 15-20.

Kodrík D., Socha R. \& Syrová Z. 2003: Developmental and diel changes of adipokinetic hormone in CNS and haemolymph of the flightless wing-polymorphic bug, Pyrrhocoris apterus (L.). J. Insect Physiol. 49: 53-61.

Kubli E. 2003: Sex-peptides: seminal peptides of the Drosophila male. Cell. Mol. Life Sci. 60: 1689-1704.

LOHER W. 1961: The chemical acceleration of the maturation process and its hormonal control in the male of the desert locust. Proc. R. Soc. (B) 153: 380-397.

Maxová A., Kodrík D., Zemek R. \& Socha R. 2001: Diel changes in adipokinetic response and walking activity of Pyr- 
rhocoris apterus (L.) (Heteroptera) in relation to physiological status and wing dimorphism. Eur. J. Entomol. 98: 433-438.

Mulye H. \& Davey K.G. 1995: The feeding stimulus in Rhodnius prolixus is transmitted to the brain by a humoral factor. J. Exp. Biol. 198: 1087-1092.

Osorio S., Piulachs M.-D. \& Bellés X. 1998: Feeding and activation of corpora allata in the cockroach Blattella germanica (L.) (Dictyoptera, Blattellidae). J. Insect Physiol. 44: 31-38.

Pener M.P. 1968: The effect of corpora allata on sexual behaviour and "adult diapause" in males of the red locust. Entomol. Exp. Appl. 11: 94-100.

RANKIN M.A. \& RIDDIFORD L.M. 1977: Hormonal control of migratory flight in Oncopeltus fasciatus: The effects of the corpus cardiacum, corpus allatum, and starvation on migration and reproduction. Gen. Comp. Endocr. 33: 309-321.

SLÁmA K. 1964: Hormonal control of respiratory metabolism during growth, reproduction, and diapause in male adults of Pyrrhocoris apterus L. (Hemiptera). Biol. Bull. 127: 499-510.

SochA R. 1993: Pyrrhocoris apterus (Heteroptera) - an experimental model species: a review. Eur. J. Entomol. 90: 241-286.

SocHA R. 2001: Latitudinal gradient in response of wing polymorphism to photoperiod in a flightless bug, Pyrrhocoris apterus (Heteroptera: Pyrrhocoridae). Eur. J. Entomol. 98: $167-169$.

SocHA R. 2004: Decreased mating propensity of macropterous morph in a flightless wing-polymorphic insect, Pyrrhocoris apterus (L.) (Heteroptera). Eur. J. Entomol. 101: 539-545.

SocHA R. 2006: Endocrine control of wing morph-related difference in mating success and accessory gland size in the firebug males. Anim. Behav. (in press).

Socha R. \& Kodrík D. 1999: Differences in adipokinetic responses of Pyrrhocoris apterus (Heteroptera) in relation to wing dimorphism and diapause. Physiol. Entomol. 24: 278-284.

Socha R. \& Šula J. 1996: Differences in haemolymph proteins in relation to diapause and wing dimorphism in Pyrrhocoris apterus (L.) (Heteroptera: Pyrrhocoridae). J. Comp. Physiol. (B) 166: 382-387.

Socha R. \& ZemeK R. 2000a: Wing movement behavior in longand short-winged morphs of flightless bug Pyrrhocoris apterus L. (Heteroptera: Pyrrhocoridae). J. Insect Behav. 13: $741-750$.

Socha R. \& ZemeK R. 2000b: Locomotor activity in adult Pyrrhocoris apterus (Heteroptera) in relation to sex, physiological status and wing dimorphism. Physiol. Entomol. 25: 383-389.

Socha R. \& ZEMEK R. 2003: Wing morph-related differences in the walking pattern and dispersal in a flightless bug, Pyrrhocoris apterus (L.) (Heteroptera). Oikos 100: 35-43.

SochA R. \& ZEMEK R. 2004a: Mating behaviour and wing morph-related differences in the sexual activity of a flightless bug, Pyrrhocoris apterus (L.) (Heteroptera). Ethol. Ecol. Evol. 16: $217-229$.

Sосна R. \& Zемек R. 2004b: Wing morph-related differences in the pattern of mating activity in a flightless bug, Pyrrhocoris apterus (L.) (Heteroptera). Inv. Repr. Dev. 46: 11-18.

Socha R., Šula J. \& Zemek R. 1997: Feeding, drinking and digestive enzyme activities in long- and short-day females of Pyrrhocoris apterus (Heteroptera). Physiol. Entomol. 22: 161-169.

Socha R., Šula J. \& Zemek R. 1998: Feeding behaviour, digestive physiology and lipid content in macropterous females of Pyrrhocoris apterus (L.) (Heteroptera: Pyrrhocoridae). Physiol. Entomol. 23: 91-96.

Socha R., Kodrík D. \& Zemek R. 1999: Adipokinetic hormone stimulates insect locomotor activity. Naturwissenschaften $\mathbf{8 8}$ : 383-389.

Socha R., Ś ULA J. \& KodRíK D. 2001: Sexual activity in macropterous and brachypterous males of a flightless bug, Pyrrhocoris apterus (Heteroptera). Eur. J. Entomol. 98: 19-24.

Socha R., ŠUla J. \& Kodrík D. 2004: Wing morph-related differences in developmental pattern of accessory gland proteins in adult males of Pyrrhocoris apterus (L.) and their endocrine control. J. Insect Physiol. 50: 893-901.

Socha R., Kodrík D. \& Šula J. 2005: Wing morph-specific differences in the metabolism and endocrine control of reserve mobilization in adult males of a flightless bug, Pyrrhocoris apterus (L.), Heteroptera. J. Comp. Physiol. (B) 175: 557-565.

TANAKA S. 1999: Resource allocation, metabolic rate and hormonal control of the reproductive organs and flight muscle in male adults of a wing dimorphic cricket, Modicogryllus confirmatus. Entomol. Sci. 2: 315-327.

WAJC E. \& Pener M.P. 1969: The effect of the corpora allata on the mating behavior of the male migratory locust, Locusta migratoria migratorioides (R. \& F.). Israel J. Zool. 18: 179-192.

Wilson T.G., De Moor S. \& Lei J. 2003: Juvenile hormone involvement in Drosophila melanogaster male reproduction as suggested by the Methoprene-tolerant ${ }^{27}$ mutant phenotype. Insect Biochem. Mol. Biol. 33: 1167-1175.

WOLFNER M.F. 2002: The gifts that keep on giving: physiological functions and evolutionary dynamics of male seminal proteins in Drosophila. Heredity 88: 85-93.

Zera A.J. \& Denno R.F. 1997: Physiology and ecology of dispersal polymorphism in insects. Annu. Rev. Entomol. 42: 207-231.

ŽĎÁREK J. 1970: Mating behaviour in the bug, Pyrrhocoris apterus L. (Heteroptera): ontogeny and its environmental control. Behaviour 37: 253-268.

Received May 25, 2005; revised and accepted September 19, 2005 